\title{
A Recent Review on Chemiluminescence Reaction, Principle and Application on Pharmaceutical Analysis
}

\author{
Tadesse Haile Fereja, ${ }^{1}$ Ariaya Hymete, ${ }^{2}$ and Thirumurugan Gunasekaran ${ }^{1}$ \\ ${ }^{1}$ Department of Pharmacy, College of Medicine and Health Sciences, Ambo University, Ambo 19, Ethiopia \\ ${ }^{2}$ Department of Pharmaceutical Chemistry and Pharmacognosy, Addis Ababa University, Addis Ababa 117, Ethiopia \\ Correspondence should be addressed to Tadesse Haile Fereja; tdss_haile@yahoo.com
}

Received 16 July 2013; Accepted 15 September 2013

Academic Editors: V. M. Bermudez, H. J. Byrne, and Z. Wang

Copyright (c) 2013 Tadesse Haile Fereja et al. This is an open access article distributed under the Creative Commons Attribution License, which permits unrestricted use, distribution, and reproduction in any medium, provided the original work is properly cited.

This paper provides a general review on principle of chemiluminescent reactions and their recent applications in drug analysis. The structural requirements for chemiluminescent reactions and the different factors that affect the efficiency of analysis are included in the review. Chemiluminescence application in immunoassay is the new version for this review. Practical considerations are not included in the review since the main interest is to state, through the aforementioned applications, that chemiluminescence has been, is, and will be a versatile tool for pharmaceutical analysis in future years.

\section{Introduction}

Luminescence is the most conveniently defined as the radiation emitted by a molecule, or an atom, when these species return to the ground state from the exited state. According to the source of excitation, luminescence phenomenon could be classified as photoluminescence (fluorescence and phosphorescence) when the excitation source is energy from absorbed light, chemiluminescence-energy from chemical reactions and bioluminescence energy from biologically catalysed reactions. An exited molecule has the same geometry and is in the same environment as it was in ground state. In this situation it either emits a photon from the same vibrational level to which it was exited initially or it undergoes in vibrational level prior to emission of radiation $[1,2]$. For an isolated molecule in the gas phase, the only way to lose vibrational energy is to emit an infrared photon, which is less probable than undergoing an electronic transition to return to the ground state. Therefore, one tends to see photon emission from higher vibrational levels of exited states in gas phase spectra at low pressure. In a solution, however, thermal relaxation of a vibrationally exited molecule is quite rapid through transfer of excess vibrational energy from the solute molecule to the solvent. In fact, this process is so efficient that all the excess vibrational energy of the exited state is lost, and this process occurs in $10^{-13}$ to $10^{-11} \mathrm{sec}$. This means that before an exited molecule in a solution can emit a photon, it will undergo vibrational relaxation, and therefore photon emission will always occur from the lowest vibrational level of an excited state $[3,4]$.

Once a molecule arrives at the lowest vibrational level of an exited singlet state, it can do a number of things, one of which is to return to the ground state by photon emission. This process is called fluorescence. The lifetime pf an exited singlet state is approximately $10^{-9}$ to $10^{-7} \mathrm{sec}$ and therefore the decay time of fluorescence is of the same order of magnitude. The quantum efficiency of fluorescence is defined as the fraction of molecules that will fluoresce.

In addition to fluorescence, one also encounters radiationless process where molecules in an excited singlet state may return to the ground state without the emission of a photon, converting all the excitation energy into heat: the process called internal conversion. Generally, internal conversion is an inefficient process and is probably only a small fraction of the total excitation energy in most molecules. Although population of triplet states by direct absorption from the ground state is insignificant, a more efficient process exists for population of triplet states from the lowest excited singlet state in many molecules. This process is referred 


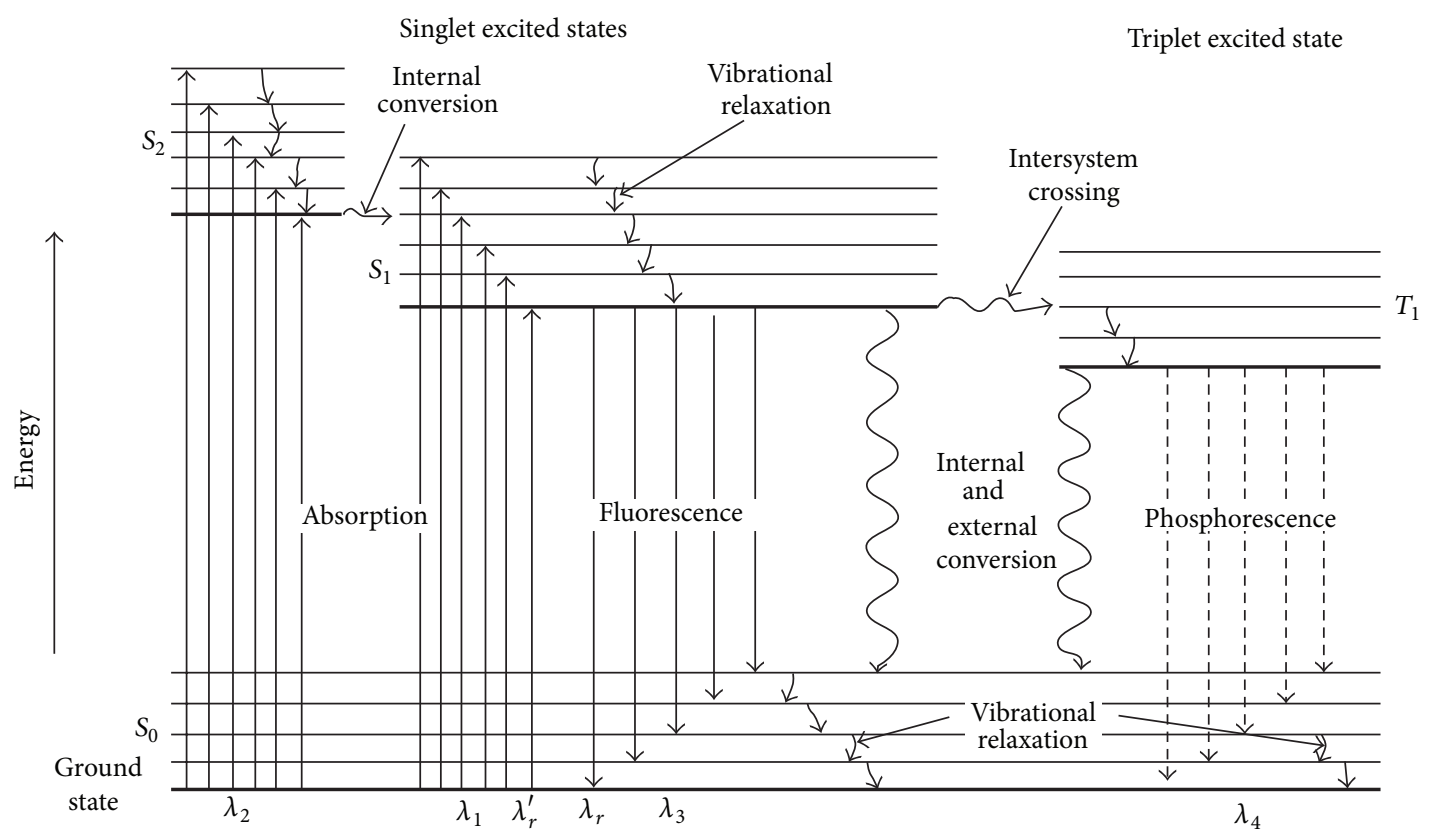

FIGURE 1: Jablonski diagram describing the electronic levels of common organic molecules and possible transitions between different singlet and triplet states. (M. Sauer, J. Hofkens, and J. Enderlein. Handbook of Fluorescence Spectroscopy and Imaging).

to as intersystem crossing and is a spin-dependent internal conversion process. A molecule in excited triplet state may not always use intersystem crossing to return to the ground state. It could loss energy by emission of a photon: the process called phosphorescence. A triplet-singlet transition is much less probable than a singlet-singlet transition. The lifetime of the excited triplet state can be up to $10 \mathrm{sec}$, in comparison with $10^{-5} \mathrm{~s}$ to $10^{-9} \mathrm{~s}$ of average lifetime of an excited singlet state. Emission from triplet-singlet transition can continue after initial irradiation $[19,20]$.

\section{The Nature of Chemiluminescence Reactions}

In general, chemiluminescence reaction yields one of the reaction products in an electronic excited state producing light on falling to the ground state. As can be seen in Figure 1, the process of light emission in chemiluminescence is the same as in photoluminescence, except for the excitation process. In fluorescence and phosphorescence, the electronically excited state is produced by absorption of Uv-visible light returning to the ground state $\left(\mathrm{S}_{0}\right)$ from the lowest singlet excited state $\left(S_{1}\right)$ or from the triplet excited state $\left(T_{1}\right)[21,22]$.

In general, a chemiluminescent reaction can be generated by two basic mechanisms (Figure 2) in a direct reaction, two reagents, usually a substrate and an oxidant in the presence of some cofactors, react to from a product or intermediate, sometimes in the presence of a catalyst. Then some fraction of the product or intermediate will be formed in an electronically excited state, which can subsequently relax to the ground state with emission of a photon. The substrate is the CL, precursors, which is converted into the electronically excited molecule, responsible for light emission or acting as the energy as transfer donor in indirect CL. The catalyst enzyme or metal ions reduce the activation energy and provide an adequate environment for producing high CL efficiency out of the process. Cofactors sometimes are necessary to convert one or more of the substrates into a form capable of reacting and interacting with the catalyst, or to provide an efficient leaving group if bond cleavage is required to produce the excited emitter. On the contrary, indirect or sensitized CL is based on a process of transfer of energy of the excited specie's to a fluorophore [23]. This process makes it possible for those molecules that are unable to be directly involved in CL reaction to transfer their excess of energy to a fluorophore that in turn is excited, releasing to its ground state with photon emission. All of these paths lead to a great variety of practical uses of CL in solid, gas, and liquid phases [24].

2.1. Requirements for Chemiluminescence Emission. For a chemical reaction to produce light, it should meet some essential requirements.

(1) The reaction must be exothermic to produce sufficient energy to form the electronically excited state. To predict whether the CL reaction will occur or not, it is possible to use free energy $(\Delta G)$ :

$$
\Delta G=\Delta H-T \Delta S
$$

where $T$ is temperature, $\Delta H$ is enthalpy, and $\Delta S$ is entropy. 


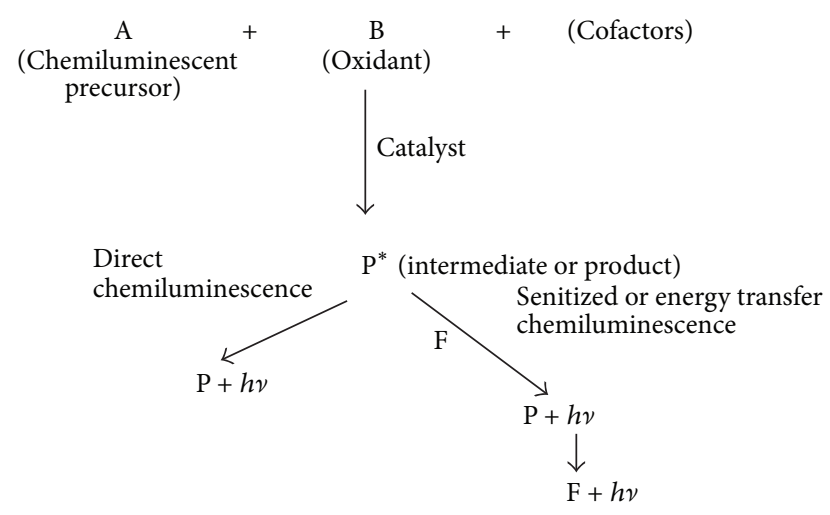

Figure 2: Types of CL reaction, $\mathrm{P}$, product $\mathrm{F}$, fluorescing substance.

Enthalpy is the actual energy source for generating the electronically excited state product. To initiate the chemical reaction, the activation energy $\left(\Delta H_{A}\right)$ is absorbed. The available energy to produce the excited state $\left(\Delta E_{\mathrm{EX}}\right)$ must be the difference between the reaction energy and the activation energy. If the difference is equal to or greater than the energy required for generating the excited state $\Delta E_{\mathrm{EX}}$, the CL process will be produced. Consider

$$
\text { Energy Available }=\Delta H_{A}-\Delta H_{E} \geq \Delta E_{\mathrm{EX}}
$$

In many CL reactions, the entropy change is small, so $\Delta G$ and $\Delta H$ are very similar in magnitude and the energetic requirements can be established in terms of $\left(\mathrm{Kcal}_{\mathrm{mol}}{ }^{-1}\right)$. In this sense, for CL to occur, the reaction must be sufficiently exothermic such that

$$
\Delta G \geq \frac{h c}{\lambda_{\mathrm{ex}}}=\frac{2.86 \times 10^{4}}{\lambda_{\mathrm{ex}}}
$$

where $\lambda_{\text {ex }}$ is the long wavelength limit (nanometers) for excitation of the luminescent species. Because most of the $\mathrm{CL}$ reaction produce photons in the range 400 (violet)-750 (red) nm, the creation of the electronically excited state and the generation of CL in the visible region require around $40-70 \mathrm{Kcal} \cdot \mathrm{mol}^{-1}$. This exothermic condition is associated with redox reactions using oxygen and hydrogen peroxide or similar potential oxidants [25].

(2) The reaction pathway must be favorable to channel the energy for the formation of an electronically excited state. In case the chemical energy is lost as heat, for example via vibration and rotational energy ways, the reaction will not be chemiluminescent.

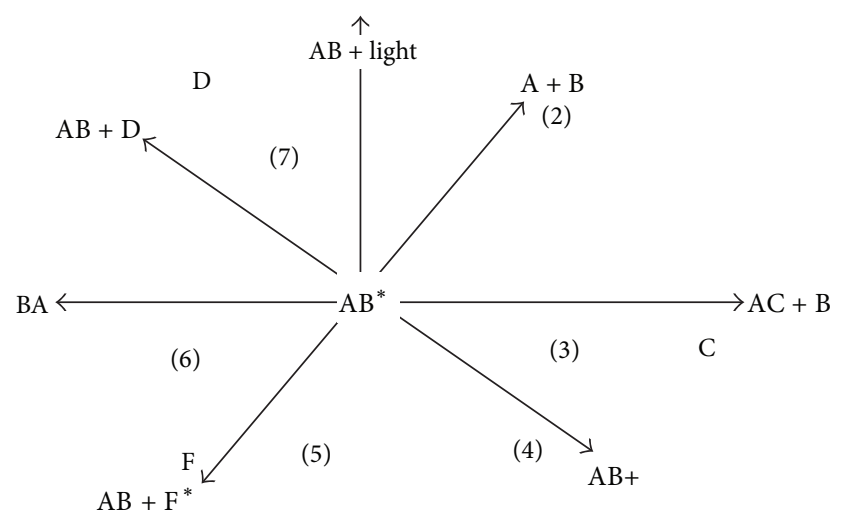

FIGURE 3: different processes for losing energy from the excited state: (1) direct CL, (2) molecules dissociation; (3) chemical reaction with other species, (4) intramolecular energy transfer, (5) intermolecular energy transfer (in case of a fluorphore, indirect CL), (6) isomerization, and (7) physical quenching.

(3) Photon emission must be a favorable deactivation process of the excited product in relation to other competitive nonradiative processes that may appear in low proportion (Figure 3 ) in the case of sensitized CL, both the efficiency of energy transfer from the excited species to the fluorophore and the fluroesence efficiency of the latter must be important.

In all the luminescent process, the intensity of the produced emission depends on the efficiency of generating molecules in the excited state, which is represented by the quantum efficiency (quantum yield) and the rate of the reaction. In the case of CL reactions, the intensity can be expressed as

$$
I_{\mathrm{CL}}=\dot{\emptyset}_{\mathrm{CL}}-\frac{\mathrm{d} A}{\mathrm{~d} t}
$$

where $I_{\mathrm{CL}}$ is the CL emission intensity (photons/second) $\emptyset_{\mathrm{CL}}$ is the CL quantum yield and $(-\mathrm{d} A / \mathrm{d} t)$ is the rate at which the CL precursor $A$ is consumed. Higher values of quantum yields are usually associated with $\mathrm{BL}$ reactions whereas in most of the CL reactions used for analytical purposes range from 0.001 to 0.1 and even very inefficient systems with much lower quantum yields can be used in analysis based on the complete absence of background emission. This is the case with ultraweak CL reactions, in which can be less than 0.001 (often $10^{-3}-10^{-2}$ ) and sometimes $10^{-9}-10^{-15}$.

Ultra-weak CL is produced from oxidative reaction in living cells and the emitted signals are mostly $10^{3}-10^{6}$ times less intense than those from luminous organisms. The main characteristic of ultraweak CL emission is that it is invisible to the naked eye. This kind of CL includes a group of CL reactions that at least in living cells, involve a number of oxygen intermediate that play an important role in certain types of cell activation in the defense system of the body, and in ischemic heart diseases. It has been detected from a wide variety of infact organs, isolated cells, and tissue homogenates from vertebrates, invertebrates, plants, and from several 
reactions in vitro. Ultra-weak CL is associated with some important cellular functions, such as mitochondrial respirations, photosynthesis, cell division, or phagocytosis, among others [26-28].

\subsection{Some Factors Influencing Chemiluminescence's Emission.} Because of the cited dependence of CL intensity upon various parameter, CL measurement's are strongly modified by experimental factors that affect quantum yield and rate of reaction, such as

(i) the chemical structure of the CL precursor, not only the central portion containing the electronically excited group, but also the side chain;

(ii) the nature and concentration of other substrates affecting the CL pathway and favoring other nonradiative competition process;

(iii) the hydrophobicity of the solvent and solution composition as example the of luminal oxidized in dimethylusfoxide (DMSO) is 0.05 compared with 0.01 in water, the colors being blue-violet $(425 \mathrm{~nm})$ and blue green (480-502 $\mathrm{nm}$ ), respectively;

(iv) the presence of energy transfer acceptors [29].

2.2.1. Chemical Considerations. A sine qua non for CL to occur is that the precursor(s) of the light-emitting species must participate in reaction that releases a considerable amount of energy. For visible emission (say 400-750 nm in wavelength), $40-70 \mathrm{kcal} \cdot \mathrm{mol}$ is required. Actually, the enthalpies of reaction have to be slightly greater than these values owing to energy losses by thermal relaxation in the ground state (after emission) and lowest excited singlet (after excitation) states of the fluorophore. Normally only certain oxidation-reduction reactions generate this much energy. In addition, at least some of the energy produced must be channeled into a reactions pathway, in which at least one of the upper vibration levels of the reactants, probably corresponding to the transition state of the initial reaction, has the same energy and a comparable geometrical structure as an upper vibrational level of the lowest excited singlet state of potentially emissive product of the reaction. The geometric identity or near-identity of the interconverting species ensures that most of the energy of activation is channeled into free energy of activation rather than being wasted as entropy or activation [30,31].

Invariably, there will also be pathway for dark reaction. The dark reaction may lead directly to the ground, electronic state species that also results from the fluorescence of the excited product, but this is not necessarily so. It is possible to have a competing dark reaction that leads directly to ground state products different from that produced by fluorescence. If $\Delta H_{4}$, is the sum of the enthalpies of activation for all dark reaction competing with the chemilluminercent pathway, whose enthalpy of activation is $\Delta H_{4 i}$ CL will be probable when $\Delta H_{4}<\Delta H_{4 i}(3,8)$. This occurs when the lowest excited singlet state of the flurophore has the same geometrical configuration as the ground electronic state of the reactants

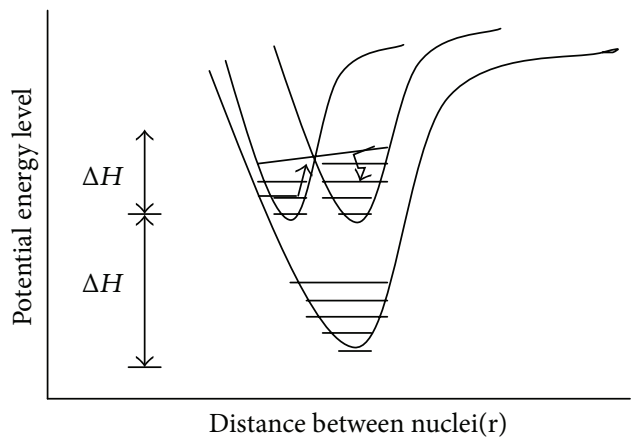

FIGURE 4: Relative positions of the potential energy $(E)$ surface of the electronic states involved in a hypothetical chemiluminescent reaction as a function of internuclear separation $(r)$.

at lower energy than when the dark product in the ground electronic state hase the same configuration as the reactants in their ground electronics state. This is illustrated in Figure 4. Often, catalysts, which are usually transition metal ions in chemilluminercent reaction and enzymes in bioluminescent reactions, are employed to make light emitting reactions processed at a convenient rate. This is accomplished by the catalyst forming a transient complex with reagents. Whose $\Delta H_{4 i}$, is lower than that of unanalyzed reaction. The catalyst is usually a cooxidant; that is, it assists electron transfer. The catalysis of the luminol hydrogen peroxide reaction by $\mathrm{Co}(\mathrm{II})$ and of the luciferian-oxygen reaction by the enzyme luciferase are important examples $(6,13)$.

$p$ and $p^{*}$ represent the ground and lowest electronically excited singlet states of the product of the reaction, respectively, and $R$ represents the ground electronic state of the reactant. $\Delta H$ is the enthalpy of the "dark" reaction while $\Delta H_{4}$ is the enthalpy of activation. $\Delta H_{4}$ is the enthalpy of activation of the photoreaction $h v$ that denotes the emission of chemiluminescence [32].

2.2.2. Photophysical Considerations. Subsequent to the formation of the potentially CL molecule in its lowest excited singlet state, a series of events carry the excited molecule down to its ground electronic state. Since the electronically excited molecule is initially also vibrationally excited, rapid, stepwise $\left(10^{-13}-10^{-12} \mathrm{~s}\right)$ thermal deactivation of the excited molecule, called thermal or vibrational relaxation, in which the molecule loses vibrational energy by inelastic collisions with the solvent occurs. The process of vibrational relaxation carries the molecule to the lowest vibrational level of the lowest excited singlet state. Certain molecules may return radiationlesly all the way to the ground electronic state in the same time frame by thermal deactivation, a process known as internal conversion. However, in some molecules, for a variety of reactions, the return from the lowest vibrational level of the lowest excited singlet state to the lowest vibrational level of the ground state by internal conversation and vibrational relaxation is forbidden (i.e., of low probability or long duration). In these molecules, return to the ground electronic 
state occurs by one of two alternative pathways, the simpler being the direct emission of ultraviolet or visible radiation whose frequency or wavelength is governed by the energy gap between the lowest excited single state and the ground electronic state [33]. The radiative transition between excited and ground state of the same spin multiplicity occurs in a time frame $10^{-11}-10^{-7} \mathrm{~s}$ after excitation, and is called fluorescence. Owing to the fact that the ground electronic state of molecule has several vibrational levels associated with it, fluorescence emission does not occur at a single wavelength but rather over a range of wavelength corresponding to several vibrational transitions as components of a single electronic transition [34].

Several processes may compete with fluorescence for deactivation of the lowest excited singlet state. As a result, only a fraction of the molecules formed in the lowest excited singlet state. $\varnothing$ Actually fluoresce. $\varnothing$ is called the quantum yield or fluorescence efficiency. It is usually a fraction but may be unity in some exceptional cases and is related to the probabilities (rate constant) of fluorescence $\left(k_{\mathrm{ef}}\right)$ and competitive process $\left(k_{d}\right)$ [35].

\subsubsection{Structural and Environmental Consideration.}

Molecules that enter into CL reaction are generally reduced species that can be easily oxidized. Molecules containing amino and hydroxyl groups fall into this category, as do polycyclic aromatic ring systems. Under strongly alkaline conditions, hydroxyl groups and even some arylamino or arylamido groups can be deprotonated making them even more susceptible to oxidation (Table 1). On the other hand, electron-withdrawing groups tend to stabilize electronic charge and make oxidation more difficult in molecules to which they are affixed. Form the chemical point of view, the solvent in which the CL experiment is carried out can have a dramatic influence on the efficiency of the $\mathrm{CL}$ reaction as salvation can alter the shapes, the depths, and the densities of the vibrational states of the potential surfaces representing the ground states of products and reactants and the lowest excited singlet state of the potential fluorophore. The alteration of the intersections of these potential energy surface an affect the enthalpies of reaction and the enthalpies of activation for dark and lumigenic reactions. In some cases, these changes will favor CL (if $\Delta H^{*}$ decreases relative to $\Delta H^{*}$ ) and in some cases, they will make it thermodynamically unfavorable for CL to occur. Other influences of the structure and the environment are manifest in the rates of processes competing with fluorescence for deactivation of the lowest excited singlet state. These are the process and properties that influence the fluorescence process $[36,37]$.

\subsubsection{The Influence of Molecular Structure on Fluorescence.} Among other factors, the quantum yield of fluorescence determines the intensity of lights emission in a CL. This, as well as the position in the spectrum occupied by the fluorescence band, is largely a function of the molecular structure. Fluorescence is most often observed in highly conjugated or aromatic organic molecules with rigid molecular skeletons.
The less vibrational and rotational freedom that the molecules has, the greater is the probability that the energy gap between the lowest excited singlet state and the ground electronic state will be large, so that fluorescence will predominate over nonradiative deactivation and assure a high fluorescence efficiency.

Aromatic molecules containing freely rotating substituents usually tend to fluoresce less intensely than those without these substituents. These result from the introduction into each electronics state of rotational and vibrational substates by the exocyclic substituents [37].

\subsubsection{The Influence of the Environment on the Fluorescence Spectrum}

Solvent Effect. Solvent interactions with molecules are mainly electronic and it is usually the difference between the electrostatic stabilization energies of ground and excited state that contribute to the relative intensities and spectral positions of fluorescence in different solvents. The change in electron distribution that takes place upon transition from the lowest excited singlet state to the ground state during fluorescence causes changes in the dipolar and hydrogenbonding properties of the solute. If the solute is more polar in the excited state than in the ground state, fluorescence will occur at longer wavelength in a polar solvent than in a nonpolar solvent. This is because, the more polar solvent will stabilize the excited state relative to the ground state. Moreover, the fact that photoluminescence originates from an excited state that is in equilibrium with its solvent cage and terminates in a ground state that is not cause the fluorescence to lie at longer wavelengths the more polar or more strongly hydrogen-bonding the solvent [38].

The lowest excited singlet state is formed by promoting a lone-pair electron to a vacant $\pi$ orbital (this is called an $n, \pi^{*}$ state). These molecules tend to show very little fluorescence in aprotic solvents such as aliphatic hydrocarbons because the $n, \pi^{*}$ excited singlet state is efficiently deactivated by intersystem crossing. However, in portic solvents such as water to ethanol, these molecules become fluorescent. This results from the destabilization of the lowest $\operatorname{singlet} n, \pi^{*}$ state by hydrogen bonding. If this interaction is sufficiently strong, the fluorescent $\pi, \pi^{*}$ state drops below the $n, \pi^{*}$ state allowing intense fluorescence. Quinoline, for example, fluoresces in water but not in cyclohexane [39].

Solvents containing atoms of high atomic number (e.g., alky iodide or bromides) also have a substantial effect on the intensity of fluorescence of solute molecules. Atoms of high atomic number in the solvent cage of the solute molecule enhance spin-orbital coupling in the lowest excited singlet state of the solute. This favors the radiationless population of the lowest triplet state at the expense of the lowest excited singlet state. Thus in heavy-atom solvents, all the other things being equal, fluorescence is always less intense than that in solvent of low molecular weight [40].

Quenching of Fluorescence. Fluorescence may be decreased or completely eliminated by interactions with other chemical 
TABLE 1: Common application of the main CL System.

\begin{tabular}{|c|c|c|c|}
\hline Phase & Reagents & Analyte & References \\
\hline Gas & Ethylene & $\mathrm{O}_{3}$ & {$[5]$} \\
\hline Gas & $\begin{array}{l}\mathrm{O}_{3} \text { after conversion of the analyte } \\
\text { to } \mathrm{NO}\end{array}$ & Nitrosamine total nitrogen & {$[6]$} \\
\hline Gas & $\mathrm{H}_{2}$ flame & Sulfer compounds & {$[7]$} \\
\hline Gas & $\mathrm{H}_{2}$ flame followed by $\mathrm{O}_{3}$ & Sulfer compounds & {$[7]$} \\
\hline Liquid & Luminal and derivatives & $\begin{array}{l}\text { Metal ions and complexes }(\mathrm{Co}(\mathrm{II}), \mathrm{Cu}(\mathrm{II}), \mathrm{Fe}(\mathrm{III}), \mathrm{Zn}(\mathrm{I}), \mathrm{Cd}(\mathrm{II}) \text {, } \\
\mathrm{Mn}(\mathrm{II}), \mathrm{Cr}(\mathrm{III}), \mathrm{Cr}(\mathrm{Iv}), \mathrm{Pt}(\mathrm{Iv}), \mathrm{ClO}^{-} \mathrm{Fe}(\mathrm{CN})_{6}^{-3}, \\
\text { Heme compounds, Peroxides, oxidants }\left(\mathrm{H}_{2} \mathrm{O}_{2}, \mathrm{O}_{2}, \mathrm{I}_{2} \text { etc...), }\right. \\
\text { Inhibitors }(\mathrm{Ag}(\mathrm{I}), \mathrm{Ce}(\mathrm{IV}), \mathrm{Ti}(\mathrm{IV}) \\
\text { Substance easily oxidized and indirectly determined (Ascorbic acid, } \\
\text { carboxylic acids, amines, etc...) } \\
\text { Substances converted in to } \mathrm{H}_{2} \mathrm{O}_{2} \text { (glucose, etc...) } \\
\text { Substances labeled with luminal and derivatives }\end{array}$ & {$[8-13]$} \\
\hline Liquid & Acridinium esters & $\begin{array}{l}\text { Substance labelled with acridinium esters, Ions } \mathrm{Ag}(\mathrm{I}), \mathrm{Bi}(\mathrm{III}), \mathrm{Pb}(\mathrm{II}) \text {, } \\
\mathrm{Co}(\mathrm{II}), \mathrm{Cr}(\mathrm{III}), \mathrm{Cu}(\mathrm{II}), \mathrm{Fe}(\mathrm{III}), \mathrm{Mn}((\mathrm{II}) \text {, etc...) } \\
\text { Oxidants }\left(\mathrm{H}_{2} \mathrm{O}_{2}, \mathrm{O}_{3} \text { etc. ..), Substances converted in to } \mathrm{H}_{2} \mathrm{O}_{2}\right. \\
\text { Reducing compounds }(\mathrm{Cr}(\mathrm{II}), \mathrm{Fe}(\mathrm{II}), \mathrm{Mo}(\mathrm{V}) \text {, ascorbic acids, } \\
\text { tetracyclines sugars, etc...) }\end{array}$ & {$[14,15]$} \\
\hline Liquid & Peroxyoxalates & $\begin{array}{l}\text { Oxidants }\left(\mathrm{H}_{2} \mathrm{O}_{2}, \mathrm{O}_{3} \text { etc...), Flourophores (polycyclic aromatic }\right. \\
\text { hydrocarbons, etc...), Derivativatized compounds with fluorophores } \\
\text { (amino acids, steroids, aliphatic amines, carboxylic acids, } \\
\text { catecholamines, etc...) }\end{array}$ & {$[10,12]$} \\
\hline Liquid & $\begin{array}{l}\text { Direct oxidation with } \mathrm{MnO}_{4} \text {, } \\
\mathrm{ClO}^{-}, \mathrm{Ce}(\mathrm{Iv}), \mathrm{IO}_{4}^{-}{ }^{-} \text {etc }\end{array}$ & Different molecules (usually in pharmaceutical applications) & {$[16-18]$} \\
\hline
\end{tabular}

species. This phenomenon is called quenching of fluorescence. Obviously, if the fluorescence of fluorophore generated in CL reaction is quenched the observation of chemiluminescence will be precluded.

Two kinds of quenching are distinguished. In static quenching, interaction between the potentially fluorescent molecule and the quencher takes place in the ground state, forming a nonfluorescent complex. The efficiency of quenching is governed by the formation constant of the complex as well as by the concentration of the quencher. The quenching of the fluorescence of salicylic acid by $\mathrm{Cu}(\mathrm{II})$ is an example.

In dynamic quenching (or diffusional quenching) the quenching species and the potentially fluorescent molecule react during the lifetime of the excited state of the latter. The efficiency of dynamic quenching depends upon the viscosity of the solution, the lifetime of the excited state $\left(\mathfrak{E}_{q}\right)$ of the of the luminescent species, and the concentration of the quencher [Q]. This is summarized in the Stern-Volumer equation:

$$
\frac{\varnothing}{\emptyset_{q}}=\frac{1}{1+k_{Q}\left(£_{q}\right)[Q]},
$$

where $k_{\mathrm{Q}}$ is the rate constant for encounter between quencher and potentially luminescing spices and $\varnothing$ and $\varnothing_{q}$ are the quantum yields of fluorescence in the absence and presence of concentration $[Q]$ of the quencher, respectively.

$k_{\mathrm{Q}}$ Is typical of diffusion-controlled reactions $\left(\sim 10^{10} \mathrm{M}^{-1} \mathrm{~s}^{-1}\right)$ white for a fluorescent molecule is typically $10^{-8}$ or less. Hence, $k_{q} \leq 10^{2} \mathrm{M}^{-1}$ and for dynamic quenching to be observed (say1 \% quenching), [Q] must be greater than or equal to $10^{-4} \mathrm{M}[41,42]$.

Energy Transfer. Energy transfer entails the excitation of a molecule that during the lifetime of the excited state passes its excitation energy to other molecules. The loss of excitation energy from the initial excited species (the door) results in quenching of the luminescence of the energy donor and may result in luminescence from the energy recipient (acceptor), which becomes excited in the process.

Energy transfer can occur by either or two acceptor concentration dependent processes. In the resonance excitation transfer mechanism or dipole mechanism, the donor and acceptor molecules are not in contact with one another and may be separated by as much as $10 \mathrm{~nm}$ (although transfer distance closer to $1 \mathrm{~nm}$ are more common). In the classical sense, the excited energy donor molecule may be thought of as transmitting antenna that creates an electrical field in its vicinity. Potential acceptor molecules within the range of this electrical field function as receiving antennae and absorb energy from the field resulting in their electronic excitation. The rate of resonance energy transfer decreases with the sixth power of the distance between the donor and acceptor dipoles according to

$$
K_{\mathrm{ET}}=\frac{1}{\mathfrak{E}_{D}} \frac{R_{0}}{R},
$$

where $K_{\mathrm{ET}}$ is the rate constant for resonance energy transfer, and $\mathfrak{E}_{D}$ is the lifetime of the excited state of the donor 
molecule. $R$ is the mean distance between the center for the donor and acceptor dipoles, and $R_{0}$ is constant for a given donor-acceptor pair, corresponding to the main distance between the centers of the donor and acceptor dipoles for which energy transfer from donor to acceptor and florescence from the donor are equally probable. Another general requirement for the occurrence of resonance energy transfer is the overlap of the fluorescence spectrum of the donor and the absorption spectrum of the acceptor. Any degree of overlap of these spectra will satisfy the quantization requirements for the energy or the thermally equilibrated donor molecules to promote the acceptor to a vibrational level of excited singlet state. The greater the degree or overlap of the luminescence spectrum of the donor and the absorption spectrum of the acceptor, the greater is the probability that energy transfer will take place $[43,44]$.

The exchange mechanism of excitation energy transfer is important only when the electron clouds of donor and acceptor are in direct contact. In this circumstance, the highest energy electrons of the donor and acceptor may exchange places. Thus, the optical electron of an excited donor molecule may become part of the electronic structure of an acceptor molecule originally in the ground singlet state in while the donor is returned to its ground singlet state by acquiring an electron from the acceptor. Exchange energy transfer is also the most efficient when the fluorescence spectrum of the donor overlaps the absorption spectrum of the acceptor. Exchange energy transfer is a diffusioncontrolled process (i.e., every collision between donor and acceptor leads to energy transfer) and such its rate depends on the viscosity, of the medium. Resonance energy transfer, on the other hand, is not diffusion-controlled, does not depend upon solvent viscosity and may be observed at the lowest concentrations of acceptor species. Energy transfer from the initially chemiexcited species to a suitable acceptor flowed by fluorescence from the acceptors in an important process in chemiluminescence $[45,46]$.

2.3. Chemiluminescence Reaction Parameters. CL reaction is commonly divided into two classes. In the type I (direct) reaction the oxidant and reductant interact with rate constant $k_{r}$ to directly from the excited product whose excited singlet state decays with the first (or pseuodfrist)-order rate constant $k_{r}=k_{f}+k_{d}$. In type II (indirect) reaction the oxidant and reactant interact with the formation of an initially excited product $(k)$ followed by the formation of an excited secondary product, either by subsequent chemical reaction or by energy transfer, with constant $K$. The secondary product then decays from the lowest excited singlet state with rate constant $k$. Type II reactions are generally denoted as a complex or sensitized chemiluminescence.

If $\dot{\emptyset}_{\mathrm{CL}}$ is the efficiency of the chemiluminescence reaction, which is the ratio of the number of photons emitted to the number of molecules of reactant reacting it can be defined for a type I reaction as

$$
\dot{\emptyset}_{\mathrm{CL}}=\dot{\emptyset}_{R} \times \dot{\emptyset}_{E} \times \dot{\emptyset}_{f}
$$

where $\oint_{R}$ the chemical yield, is the ratio of the number of molecules that react, through the chemilluminercent pathway to the total number of molecules reacted; $\emptyset_{E}$, the excitation yield, is the ratio the number of molecules that form an electronically excited product to the number of molecules that react through the Chemiluminescent pathway, and $\emptyset_{f}$ is the quantum yield of fluorescence of the lightemitting species.

In type II reaction

$$
\dot{\emptyset}_{\mathrm{CL}}=\dot{\emptyset}_{R} \times \dot{\emptyset}_{E} \times \dot{\emptyset}_{f} \times \dot{\emptyset}_{\mathrm{ET}},
$$

where all symbols have the same meanings as above and $\emptyset_{\mathrm{ET}}$ is the efficiency of energy transfer from the initially chemiexcited species to the energy transfer acceptor $\emptyset_{\mathrm{CL}}$, ofcourse, a function of the chemical and photo physical factors described in Section 2.2.2 such as solvent polarity, reagent concentrations, and molecular structure.

The physical significant of $\emptyset_{C L}$ is the under defined experimental conditions it is the constant of proportionality between, the observe intensity of chemiluminescence, and the rate of consumption of the initial luminophore (reactant $\mathrm{L})$; that is, $l_{\mathrm{CL}}=\emptyset_{\mathrm{CL}} \times(-\mathrm{d} L / \mathrm{d} t)[47,48]$.

\section{Chemiluminescence Based Analysis}

Because the reaction rate is function of the chemical concentration, CL techniques are suitable for quantitative analysis. The usefulness of CL system in analytical chemistry is based on some special characteristic.

(1) As the technique simultaneously comprises kinetic and luminescence features, it provides highly sensitive and wide dynamic ranges. Excellent detection limits, in the range of femtomoles, can be reached if $\emptyset_{C L}$ is high enough. As an example, in the gas phase, typical detection limits are 10 pmol NO using ozone and $0.1 \mathrm{pmol}$ for sulfur compounds using a hydrogen flame followed by ozone, in the liquid phase down to $1 \mathrm{fmol}$ of the fluorophore may be detected using the peroxyoxlalte CL system and $0.1 \mathrm{fmol}$ peroxidase when using luminol. In comparison to other spectrometric techniques, in general, CL is approximately $10^{5}$ time more sensitive than absorption spectrometry and at least $10^{3}$ times more sensitive than fluorimetry.

(2) Compared to photoluminescence process, no external light source is required, which offers some advantages such as the absence of scattering or background photoluminescence signal, the absence of problems related to instability of the external source, reduction of interferences due to a nonselective excitation process, and simple instrumentation.

(3) Selectivity and linearity are most dependants on the reaction conditions chosen. As for photoluminescence process, absorption or emission radiation by the analyte, products, or concomitants can cause nonlinearity or spectral interference. 
(4) The technique is versatile for determination of a wide variety of species that can participate in the CL process, such as CL substrates or CL precursors responsible for the excited state; the necessary reagent for the CL reaction (usually an oxidant); some species that affects the rate or efficiencies of $\mathrm{CL}$ reaction, activators such as catalyst (enzymes or metal ions) or inhibitors such as reductants that inhibit the CL emission; fluorphores in the case of sensitized CL, some species that are not directly involved in the CL reaction but that can react with other reagents in coupled reactions to generates a product that is a reactant in the $\mathrm{CL}$ reaction, species that can be derivatized with some CL precursors or fluorophores being determined by direct or sensitized CL

(5) Depending on the nature of the analyte and the CL reaction the increase or decrease of CL intensity will be directly related to the analyte concentration.

(6) CL reactions can be coupled as a detection technique in chromatography, capillary electrophoresis, or immunoassay, providing qualitative and/or quantitative information or a large variety of species in the gas and liquid phases.

(7) CL reaction can be coupled as a detection technique in chromatography, capillary electrophoresis or immunoassay, providing quantitative and/or quantitative information of a large variety of species in the gas and liquid phase.

As an empirical rule, CL behavior may be predicated in a compound or its derivatization product has fluorescence properties. It is possible that oxidization of such a species may produce CL, but there are many exceptions to this general rule [49-51].

3.1. Instrumentation. The instrumentation employed for chemiluminescence measurements basically consists of a mixing device and a detection system. Three approaches have been used to measure the intensity of emitted light.

The first approach involves the use of static measurement system in which the mixing of reagents is performed in a vessel held in front of the detector. The chemiluminescence reagent is added to the analyte in a cuvette held in a dark enclusure, and the intensity of the light emitted is measured through an adjacent photomultiplier tube. In this static system, the mixing is induced by the force of the injection. This simple reagent addition system without a mixing device is of limited usefulness in measuring fast chemiluminescent reactions with precision. The procedure is also rather cumbersome, requiring separate cuvettes for each measurements as well as repeated opening of the light-tight apparatus, which necessitates special precautions to protect the photomultiplier tube.

The second approach is the two-phase measurement system. In this system, the chemilluminercent reagents are immobilized on a solid support such as filter paper, and the analyte is permitted to interact with the immobilized reagent by diffusion or convection. The light emitted is measured using a micro liter plate reader or by contact printing with photographic detection. The chemilluminercent intensity of these systems is influenced by both the kinetics of the reaction and the efficiency of the mass transfer process bringing the reactants together. The principal advantages of this system lie in the conservation of reagents and the convenience of measurement.

The third approach involves the use of flow measurement systems. The flow injection approach has been described as the most successful of the methods. It involves injection of the analyte into a steam of appropriate $\mathrm{pH}$, remote from the detector, and the chemiluminescent reagent flows in another stream. The two streams meet at a T-junction inside a light-tight enclosure, then flow through a flat coil placed immediately in front of a photomultiplier tube. This compact assembly provides more rapid and reproducible mixing, resulting in reproducible emission intensities and permitting rapid sample through put. The design of the mixing device and the means of retaining the emitting solution in view of the detectors are of important consideration. Mixing is reported as being the most effective at a T-piece or a Y-junction, although some have used a conventional FIA in which sample is injected in to the surrounding flowing reagent to achieve mixing. This approach is reproducible but reported as not producing a rapid mixing. In flow measurement system, the chemiluminescence signal has to be measured during the mixing: as a result, only a section as opposed to the whole intensity-time curve is measured. An additional feature of these systems is that the shape of the curve now depends on both the kinetics of the chemilluminercent reaction and the parameters of the flow system. Chemical variables such as reagent concentration and $\mathrm{pH}$, and physical parameters such as flow rate, reaction coil length, sample size, and the limitations of experimental apparatus all affects the performance of flow injection procedures. The effects of these on the observed analytical signal are not necessarily independent, as interactions can and probably do occur $[52,53]$.

The sample and the reagent could be either in continuous flow or in stopped flow approach. Continues flow CL systems are used in gas phase and solution phase reactions. The sample and CL reagents are continuously pumped and mixed together using a merging zones of flow injection manifold and sent to a flow cell or mixed and the emission in an integral reactor flow cell. The signal is observed when the cell is totally filled with the reaction mixture, at a fixed period after mixing. In this case, it is possible to obtain a constant and reproducible signal, easier to measure, which represents the integrated output over the residence time of the reaction mixture in the cell. Optimum sensitivity is achieved by adjusting the flow rate, the observation cell volume, and transfer channel volumes, with the aim of observing portion of emission profile to occur at the maximum of the CL intensity- versus time profile. Disadvantage of this approach include the high reagent consumption and the fact that no kinetic information is obtained and that only a very small portion of the total CL intensity is measured for slow reactions (Figure 5).

Stopped flow approach has interesting features for CL monitoring in very fast CL reactions. In this case, the sample and reagent are efficiently mixed in a reaction chamber and 


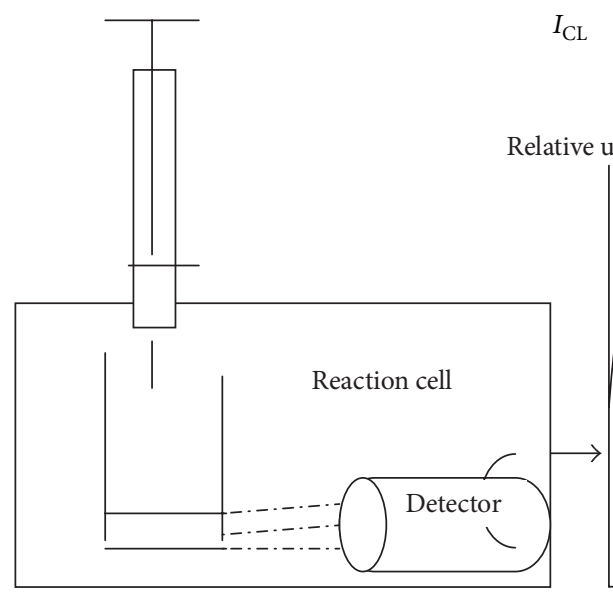

(a)

$I_{\mathrm{CL}}$

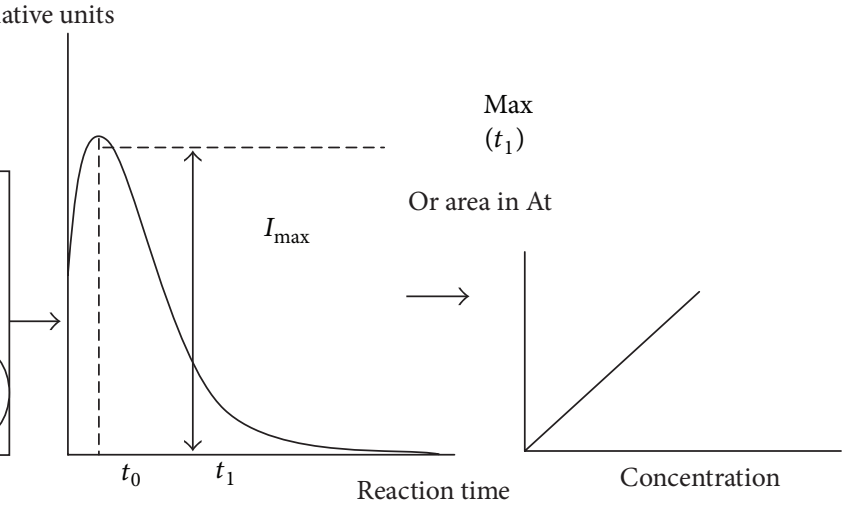

(s)

(b) (c)

FIGURE 5: Basic steps in a CL process: (a) the sample and reagent(s) are introduced is the reaction cell and the final reagent is injected to initiate the CL emission, then lights is monitored by the detector, (b) curve showing CL intensity as a function of time after reagents mixing to initiate the reaction (the decay of the signal is due to the consumption of reagents and changes in the CL quantum efficiency with time); (c) a calibration function is established in relation to increasing analyte concentrations.

rapidly forced to an observation cell, in which the flow is violently stopped, allowing monitoring of the full intensitytime curve and allowing kinetics measurements (Figure 5) which can be related to the analyte concentration with a higher precision and selectively than using peak height or areas.

3.1.1. Reaction Cell. Depending on the CL reaction that is carried out in liquid, solid or gas phases, it is possible to use different reaction cells, the essential requirements for their design being that the maximum intensity must be reached while the stream containing the analyte and the CL reagents is in front of the detector. It is a very critical variable because in both static and flow methods, the magnitude of the CL intensity is proportional to the volume of the cell. Usually it is possible to apply any material that transmits light in the visible range and that is compatible with the sample, such as glass, quartz, or even acrylic or other plastics. Polystyrene is not recommended because it can build up static electricity, which may contribute to elevated background levels. More transparent cell materials allow more light to reach the detector, resulting in better detection limits.

In the case of flow CL measurements in the liquid phase, the cell design most frequently used is a long spiral cell (about $1 \mathrm{~cm}$ ) which is located as close to the detector as possible. The large volume is required due to the need to collect a greater amount of emitted light and because the thin size required $(50-100 \mu \mathrm{m})$ minimizes band broadening and provides a thin optical path to reduce inner filtering effects of the CL emission. With the purpose of minimize sample dispersion, usually, the injection valve and the cell must be placed as close to reach the other ones as possible.

In heterogeneous systems, the reagents can be immobilized on a solid surface either covalently, behind a membrane, or by absorption, and it can interact with the analyte by diffusion or convection. In gas phase CL reaction, the sample and the CL reagents flow inside a reactor through calibrated leaks and the CL emission produced and monitored by the detector through and optical window. Commercial and homemade luminormeters for liquid, solid, and gas phase analysis use various cell configurations.

3.1.2. Detector. There are four basic requirements for the detectors. Which constitute the critical parts of the luminormeter.

(1) It must be able to detect a light signal over several orders of magnitude of intensity form just a few photons per second to tens of millions photons per second.

(2) It must be sensitive at least over the spectral range 400-600 $\mathrm{nm}$ and ideally over the complete range of the visible spectrum (i.e., 380-750 nm) and even over the UV and IR regions. When its sensitivity varies with wavelength, as is usually the case, it must be possible to correct the data in this respect.

(3) The signal output presented by the detector should be directly related to the light intensity reaching it, ideally linearly over the entire sensitively range. The signal from the detector should be produced in a form that can be easily displayed recorded and analyzed.

(4) The speed of response of the detector must be much faster than the rate of the CL reaction, if the signal output is not to be a distorted version of the true signal.

Light is detected by photosensitive devices through the generation of photocurrent. Photomultiplier tubes (PMT) are 
the detection devices more frequently used in CL because of their high gains making them suitable for low light level detection. However they must be operated with very stable power supplies to keep the total gain constant. There are two different configurations for PMTs depending on their positions in relation to the reaction cell: either to the side ("sideon" configuration) or underneath the reaction cell ("end-on" configuration). Side on PMTs is usually more economical than end on models and their vertical configuration takes up less space than be end on version. However, end-on PMTs have large photocathode area and offer greater uniformity of light collection and of the response.

Also in the absence of light stimulation all practical light detectors produce an unwanted output signal termed "background" which is referred to as "dark counts" or dark current" depending on the applications. At moderate bias, voltages, dark current can be due primarily to thermal emission of electrons at the photocathode and from the dynodes. This undesirable signal can be reduced by cooling the PMT using temperature of 60 to $0^{\circ} \mathrm{C}$.

\subsection{Pharmaceutical Application of Chemiluminescence Reac-} tion. From Table 1 one can observe that varsitile compounds and pharmaceuticals could be directly or indirectly quantified. For the indirect method of quantification either chemically derived so as to exhibit the compound luminescent effect or by measuring the compounds quenching effect.

3.3. Chemiluminescent Immunoassay. One of the most significant applications of chemiluminescent molecules has been labels in immunoassay. Not unexpectedly the constraints arise from the need to couple the chemiluminescent molecules to the analytes of respective antibodies in such a way that the properties of the label are not disturbed. In particular aminobutyl ethyl isoluminol (ABEl) which can be coupled to low molecular weight compounds without loss in quantum yield.

As a result, $\mathrm{ABEl}$ and related molecules have been used as labels for a variety of steroid immunoassays which have levels of performance comparable to those of the corresponding radioimmunoassay. A universal problem of these methods arises from the extensive quenching of the signal when the label is associated with antibody. So great is the loss in quantum yield under these conditions that dissociation of the complex prior to luminometry has been adopted as an obligatory step. As indicated above, the majority of chemiluminescent hapten immunoassays so far described have been based on ABEl or similar labels. These assays include plasma estradiol-17, testosterone, and progesterone as well as urinary conjugated steroids such as pregnanediol$3 n$-glucuronide and estrone glucuronide. Dissociation of the label prior to signal detection has been facilitated by means of strong alkali often at high temperature. Though this results in satisfactory light emission, it does introduce a step into the analytical procedure which is operationally complex and which may affect the overall performance of the assay itself. It is possible that acridinium esters will provide simpler alternatives, though as yet there is only one report of their use on steroid immunoassay. In this case a synthetic acridinium derivative of estradiol was prepared which could be detected with high sensitivity. Assay performance was however limited by the low affinity of the antibody for the labelled derivative once again illustrating the dependence of hapten systems on suitable immunochemistry.

The procedure has centred on the development of assay methods for polypeptides using acridinium ester labelled antibodies. It has employed almost exclusively two-site assay procedures in which the analyte is reacted either sequentially or simultaneously with labeled antibodies and solid-phase antibodies. In this procedure, the uptake of labelled antibody on to solid-phase antibody is a function of the quantity of analyte bound and hence the analyte concentration in the sample. The most satisfactory solid-phase antibodies developed so far are prepared by reacting antibody preparations with the diazonium salt of re-precipitated aminoaryl cellulose. This preparation has the advantage that it remains in suspension during the reaction without agitation. It is also extremely efficient as an immune adsorbent since it has a high binding capacity for protein as well as very low non-specific uptake of protein $[54,55]$.

In this procedure standards or serum samples (100 pi) are reacted with a monoclonal antibody ( $100 \mathrm{pl}, 1.5 \mathrm{ng})$ labelled with $3 \mathrm{~mol} / \mathrm{mol}$ acridinium ester. After $2 \mathrm{~h}$, solid-phase antibody suspension $(100 \mathrm{pl}, 50 \mathrm{Pg})$ is added and after a further $1 \mathrm{~h}, 1 \mathrm{~mL}$ of buffer is added and the mixture centrifuged. After one further $1 \mathrm{~mL}$ wash, the chemiluminescent activity associated with the solid-phase is quantified luminometrically following injection of $200 \mathrm{pl}$ water to resuspend the pellet followed by $200 \mathrm{p} 10.1 \%(\mathrm{v} / \mathrm{v}) \mathrm{H}_{2} \mathrm{O}_{2}$ in $0.1 \mathrm{M} \mathrm{NaOH}$. The photon counts are integrated over a $2 \mathrm{~s}$ period and the bound counts related to the dose of analyte present. The use of reagent excess methodology together with an acridinium ester label of high specific activity combine to make this the most sensitive immunoassay yet described for TSH [56, 57].

From the clinical point of view this assay offers a remarkable breakthrough in diagnostic and therapeutic testing. Pituitary TSH secretion plays a central role in the control of thyroid function. Since it is under negative feedback control by the thyroid hormones whose production it stimulates, it provides a sensitive indicator of thyroid status.

The main applications of TSH measurement being in the confirmation of hypothyroidism where levels are elevated and in monitoring the pituitary response to thyrotropin release hormone (TRH). This immune-chemiluminometric assay (ICMA) is at present under evaluation [58].

\section{References}

[1] L. Bøtter-Jensen, "Luminescence techniques: instrumentation and methods," Radiation Measurements, vol. 27, no. 5-6, pp. 749-768, 1997.

[2] Y. Hajime, Nitride Phosphors and Solid-State Lighting, Taylor \& Francis, 2011.

[3] K. H. Drexhage, "Influence of a dielectric interface on fluorescence decay time," Journal of Luminescence, vol. 1-2, no. C, pp. 693-701, 1970. 
[4] B. Wen, Thermally Stimulated Luminescence Excitation Spectroscopy as a Technique to Measure the Photoionization Energy of $\operatorname{Sr}(\mathrm{SCN})_{2}: \mathrm{Eu}^{2+}$, vol. 2004, Maureen Grasso Dean of the Graduate School, The University of Georgia.

[5] F. McElroy, EPA/NERL, Determination of Ozone by Ultraviolet Analysis, May 2000.

[6] C.-Y. Liu and A. J. Bard, "Electrochemistry and electrogenerated chemiluminescence with a single faradaic electrode," Analytical Chemistry, vol. 77, no. 16, pp. 5339-5343, 2005.

[7] A. Pavlova, P. Ivanova, and T. Dimova, "Sulfur compounds in petroleum hydrocarbon streams," Petroleum \& Coal, vol. 54, no. 1, pp. 9-13, 2012.

[8] A. A. Alwarthan and F. A. Aly, "Chemiluminescent determination of pyridoxine hydrochloride in pharmaceutical samples using flow injection," Talanta, vol. 45, no. 6, pp. 1131-1138, 1998.

[9] C. Fu-Chun, H. Yu-Ming, and Z. Zhun-Jun, "Chemiluminescent determination on a luminol reaction of streptomycin sulfate by flow injection analysis," Journal of Southwest China Normal University, vol. 2004, no. 1, pp. 79-83, 2004.

[10] J. K. Robinson, M. J. Bollinger, and J. W. Birks, "Luminol/ $\mathrm{H}_{2} \mathrm{O}_{2}$ chemiluminescence detector for the analysis of nitric oxide in exhaled breath," Analytical Chemistry, vol. 71, no. 22, pp. 51315136, 1999.

[11] H. Sun, J. Wang, P. Chen, and T. Wang, "Determination of ofioxacin and levofloxacin in pharmaceutical preparation and human urine using a new flow-injection," Asian Journal of Pharmaceutical Sciences and Research, vol. 1, no. 5, pp. 21-32, 2011.

[12] P. Thongsrisomboon, B. Liawruangrath, S. Liawruangrath, and S. Satienperakul, "Determination of nitrofurans residues in animal feeds by flow injection chemiluminescence procedure," Food Chemistry, vol. 123, no. 3, pp. 834-839, 2010.

[13] C. Yu, Y. Tang, X. Han, and X. Zheng, "Sensitive assay for catecholamines in pharmaceutical samples and blood plasma using flow injection chemiluminescence analysis," Analytical Sciences, vol. 22, no. 1, pp. 25-28, 2006.

[14] W. Freyer, C. C. Neacsu, and M. B. Raschke, "Absorption, luminescence, and Raman spectroscopic properties of thin films of benzo-annelated metal-free porphyrazines," Journal of Luminescence, vol. 128, no. 4, pp. 661-672, 2008.

[15] C. J. Borman, B. P. Sullivan, C. M. Eggleston, and P. J. S. Colberg, "The use of flow-injection analysis with chemiluminescence detection of aqueous ferrous iron in waters containing high concentrations of organic compounds," Sensors, vol. 9, no. 6, pp. 4390-4406, 2009.

[16] F. A. Aly, N. A. Alarfaff, and A. A. Alwarthan, "Permanganatebased chemiluminescence analysis of cefadroxil monohydrate in pharmaceutical samples and biological fluids using flow injection," Talanta, vol. 47, no. 2, pp. 471-478, 1998.

[17] F. A. Aly, S. A. Al-Tamimi, and A. A. Alwarthan, "Chemiluminescence determination of some fluoroquinolone derivatives in pharmaceutical formulations and biological fluids using $\left[\mathrm{Ru}\left(\right.\right.$ bipy $\left.{ }_{3}{ }^{2+}\right]-\mathrm{Ce}(\mathrm{IV})$ system," Talanta, vol. 53 , no. 4 , pp. $885-$ 893, 2001.

[18] Y.-H. Li, "Chemiluminescence determination of sulpiride in pharmaceutical preparations and biological fluids based on $\mathrm{KMnO}_{4}$-tween 80 reaction," Journal of the Chinese Chemical Society, vol. 56, no. 1, pp. 164-168, 2009.

[19] A. Penzkofer and Y. Lu, "Fluorescence quenching of rhodamine 6G in methanol at high concentration," Chemical Physics, vol. 103, no. 2-3, pp. 399-405, 1986.
[20] C. Dodeigne, L. Thunus, and R. Lejeune, "Chemiluminescence as a diagnostic tool. A review," Talanta, vol. 51, no. 3, pp. 415439, 2000.

[21] J. Hodak, D. Harvey, and B. Valeur, Introduction to Fluorescence and Phosphorescence, 2008.

[22] E. L. Wehry, "Molecular fluorescence, phosphorescence, and chemiluminescence spectrometry," Analytical Chemistry, vol. 52 , no. $5,1980$.

[23] M. Sauer, J. Hofkens, and J. Enderlein, Handbook of Fluorescence Spectroscopy and Imaging, WILEY-VCH Verlag GmbH \& Co. KGaA, Weinheim, Germany, 2011.

[24] J. Han, J. Jose, E. Mei, and K. Burgess, "Chemiluminescent energy-transfer cassettes based on fluorescein and nile red," Angewandte Chemie International Edition, vol. 46, no. 10, pp. 1684-1687, 2007.

[25] A. M. García-Campaña and W. R. G. Baeyens, "Principles and recent analytical applications of chemiluminescence," Analusis, vol. 28, no. 8, pp. 686-698, 2000.

[26] A. M. Jimenez and M. J. Navas, "Chemiluminescence methods (present and future)," Grasas y Aceites, vol. 53, no. 1, pp. 64-75, 2002.

[27] Miyawa and Schulman, "Handbook of Pharmaceutical analysis," in Luminescence Spectroscopy, pp. 457-460.

[28] R. J. Stanley, B. King, and S. G. Boxer, "Excited state energy transfer pathways in photosynthetic reaction centers. 1. Structural symmetry effects," Journal of Physical Chemistry, vol. 100, no. 29, pp. 12052-12059, 1996.

[29] F. Mccapra, The Chemiluminescence of Organic Compounds, School of Molecular Sciences, University of Sussex, Falmer, Brighton, UK.

[30] G. S. Denisov, N. S. Golubev, V. M. Schreiber, S. S. Shajakhmedov, and A. V. Shurukhina, "Effect of intermolecular hydrogen bonding and proton transfer on fluorescence of salicylic acid," Journal of Molecular Structure, vol. 436-437, pp. 153-160, 1997.

[31] H. S. Lee, Y. S. Lee, and G.-H. Jeung, "Potential energy surfaces for lith and photochemical reactions $\mathrm{Li}^{*}+\mathrm{H} 2 \leftrightarrow \mathrm{LiH}+\mathrm{H}$," Journal of Physical Chemistry A, vol. 103, no. 50, pp. 11080-11088, 1999.

[32] M. Anpo and M. Che, "Applications of photoluminescence techniques to the characterization of solid surfaces in relation to adsorption, catalysis, and photocatalysis," Advances in Catalysis, vol. 44, pp. 119-257, 1999.

[33] C. Jan Hummelen, T. M. Luider, D. Oudman, J. N. Koek, and $\mathrm{H}$. Wynberg, 1,2-Dioxetanes: Luminescent and Nonluminescent Decomposition, Chemistry, and Potential Applications, State University of Groningen, Groningen, The Netherlands.

[34] E. F. Fabrizio, A. Payne, N. E. Westlund, A. J. Bard, and P. P. Magnus, "Photophysical, electrochemical, and electrogenerated chemiluminescent properties of 9,10-dimethyl-7,12diphenylbenzo[k]fluoranthene and 9,10-dimethylsulfone-7,12diphenylbenzo[k]fluoranthene," Journal of Physical Chemistry A, vol. 106, no. 10, pp. 1961-1968, 2002.

[35] B. Valeur, D. Harvey, and J. Hodak, Molecular Fluorescence: Principles and Applications, Wiley, 2001.

[36] V. Narasimham and N. Modeling, Analysis of Chemiluminescence Sensing for Syngas, Methane and Jet-a Combustion, Georgia Institute of Technology, 2008.

[37] K. Thompson and J. Rodriguez, The Behavior of Photo-Excited Molecules: Biological Actions and Biotechnological Applications, 2008. 
[38] I. Baraldi, G. Brancolini, F. Momicchioli, G. Ponterini, and D. Vanossi, "Solvent influence on absorption and fluorescence spectra of merocyanine dyes: a theoretical and experimental study," Chemical Physics, vol. 288, no. 2-3, pp. 309-325, 2003.

[39] R.-I. Tigoianu, A. Airinep, and D.-O. Dorohoi, "Solvent influence on the electronic fluorescence spectra of anthracene," Revista de Chimie, vol. 61, no. 5, pp. 491-494, 2010.

[40] P. Hrdlovic, J. Donovalova, H. Stankovicova, and A. Gaplovsky, "Influence of polarity of solvents on the spectral properties of bichromophoric coumarins," Molecules, vol. 15, no. 12, pp. 89158932, 2010.

[41] P. Anger, P. Bharadwaj, and L. Novotny, Enhancement and Quenching of Single-Molecule Fluorescence, The American Physical Society. The Institute of Optics and Department of Physics and Astronomy, University of Rochester, Rochester, NY, USA, March 2006.

[42] A. Mark Behlke, L. Huang, L. Bogh, S. Rose, and E. J. Devor, Fluorescence Quenching by Proximal G-bases, Integrated DNA Technologies, 2005.

[43] M. Mimuro, "Visualization of excitation energy transfer processes in plants and algae," Photosynthesis Research, vol. 73, no. 1-3, pp. 133-138, 2002.

[44] D. Nolting, T. Schultz, I. V. Hertel, and R. Weinkauf, "Excited state dynamics and fragmentation channels of the protonated dipeptide H2N-Leu-Trp-COOH," Physical Chemistry Chemical Physics, vol. 8, no. 44, pp. 5247-5254, 2006.

[45] D. Kimbrough, Chemiluminescence and Anti-Oxidants, 2000.

[46] H. Sun Cho, D. H. Jeong, M.-C. Yoon et al., "Excited-state energy transfer processes in phenylene- and biphenylene-linked and directly-linked zinc(II) and free-base hybrid diporphyrins," Journal of Physical Chemistry A, vol. 105, no. 17, pp. 4200-4210, 2001.

[47] K. Zargoosh, M. J. Chaichi, S. Asghari, M. Qandalee, and M. Shamsipur, "A study of chemiluminescence from reaction of bis(2,4,6-trichlorophenyl) oxalate, hydrogen peroxide and diethyl-2-(cyclohexylamino)-5-[(E)-2-phenyl-1-ethenyl]-3,4furandicarboxylate as a novel fluorescer," Journal of the Iranian Chemical Society, vol. 7, no. 2, pp. 376-383, 2010.

[48] M. G. Prisant, C. T. Rettner, and R. N. Zare, "A direct interaction model for chemiluminescent reactions," The Journal of Chemical Physics, vol. 81, no. 6, pp. 2699-2712, 1984.

[49] M. J. Navas and A. M. Jiménez, "Chemiluminescent methods in olive oil analysis," Journal of the American Oil Chemists' Society, vol. 84, no. 5, pp. 405-411, 2007.

[50] K.-T. Wong, T.-H. Hung, T.-C. Chao, and T.-I. Ho, "Synthesis, properties, and electrogenerated chemiluminescence (ECL) of a novel carbazole-based chromophore," Tetrahedron Letters, vol. 46, no. 5, pp. 855-858, 2005.

[51] J. Yu, L. Ge, P. Dai, C. Zhan, S. Ge, and J. Huang, "A novel glucose chemiluminescence biosensor based on a rhodanine derivative chemiluminescence system and multilayer-enzyme membrane," Turkish Journal of Chemistry, vol. 34, no. 4, pp. 489-498, 2010.

[52] ACL Instruments Inc., Chemiluminescence Instrument Hardware Basic Configuration.

[53] T. Peter So and C. Y. Dong, Fluorescence Spectrophotometry, Encyclopedia of Life Sciences, Macmillan Publishers Ltd, Nature Publishing, 2002.

[54] W. R. G. Baeyens, S. G. Schulman, A. C. Calokerinos et al., "Chemiluminescence-based detection: principles and analytical applications in flowing streams and in immunoassays," Journal of Pharmaceutical and Biomedical Analysis, vol. 17, no. 6-7, pp. 941-953, 1998.

[55] C. Wang, J. Wu, C. Zong, J. Xu, and H.-X. Ju, "Chemiluminescent immunoassay and its applications," Chinese Journal of Analytical Chemistry, vol. 40, no. 1, pp. 3-10, 2012.

[56] M. Hao and Z. Ma, "An ultrasensitive chemiluminescence biosensor for carcinoembryonic antigen based on autocatalytic enlargement of immunogold nanoprobes," Sensors, vol. 12, pp. 17320-17329, 2012.

[57] W. P. Collins, Complementary Immunoassays, John Wiley \& Sons, 1988.

[58] E. Bolton and M. M. Richter, "Chemiluminescence of Tris $(2,2$ ' bipyridyl)ruthenium(II): a glowing experience," Journal of Chemical Education, vol. 78, no. 1, pp. 47-48, 2001. 

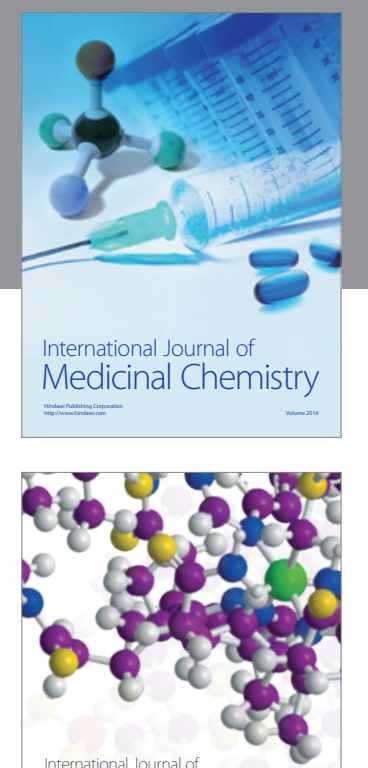

\section{Carbohydrate} Chemistry

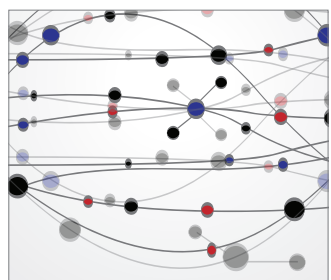

The Scientific World Journal
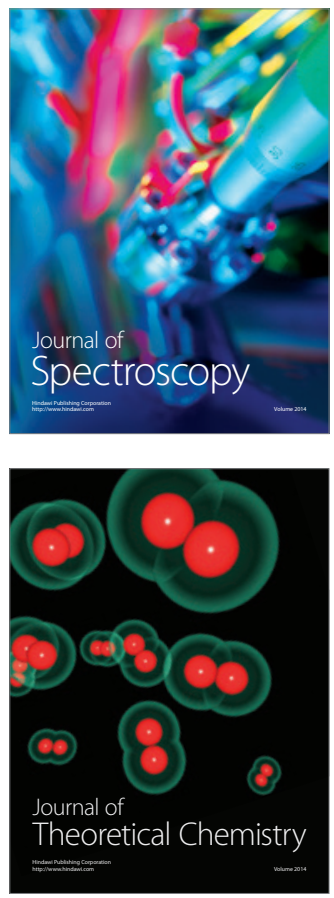
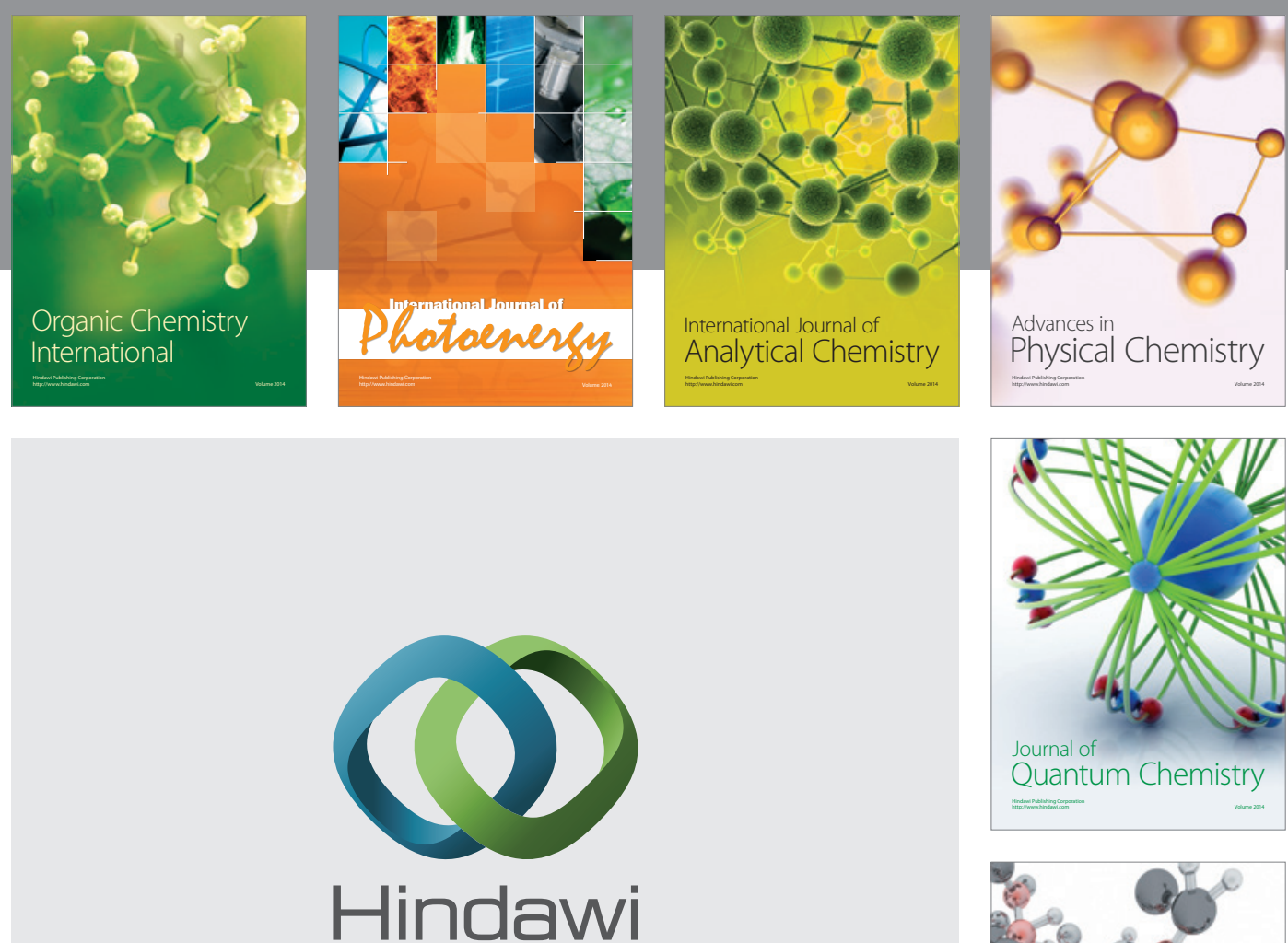

Submit your manuscripts at

http://www.hindawi.com

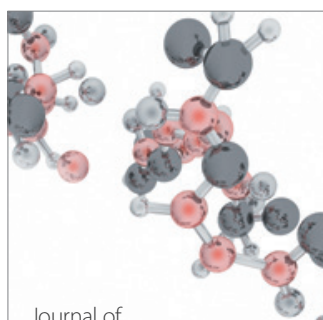

Analytical Methods

in Chemistry

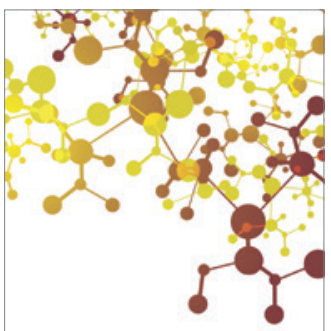

Journal of

Applied Chemistry

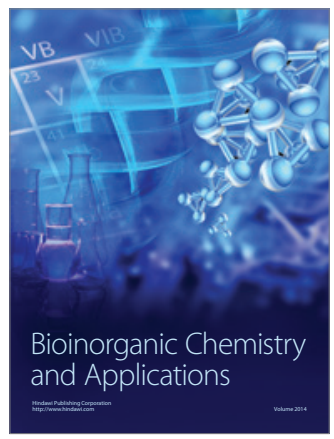

Inorganic Chemistry
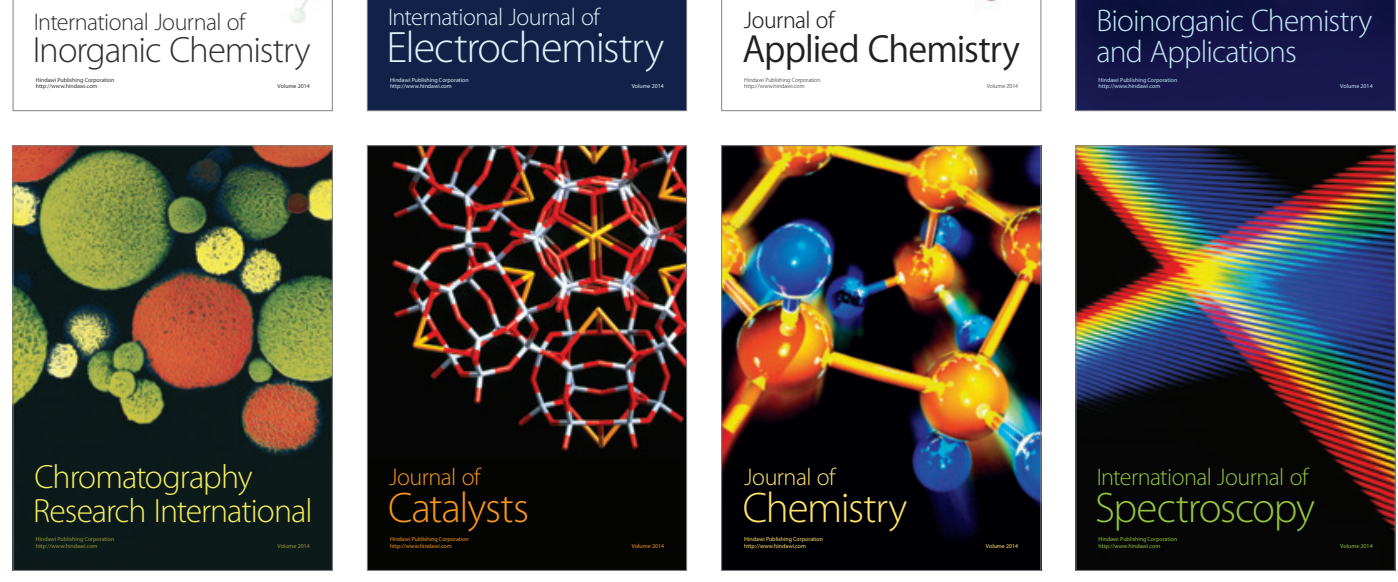